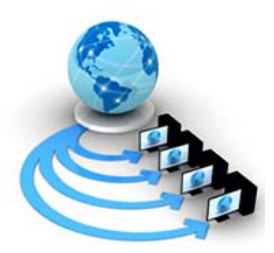

Volume 8, No. 9, November-December 2017

International Journal of Advanced Research in Computer Science

RESEARCH PAPER

\author{
Available Online at www.ijarcs.info
}

\title{
INTELLIGENT DROWSY EYE DETECTION USING CONTOURLET TRANSFORM AND WEB LOCAL DESCRIPTORS
}

\author{
Er Amrit Kaur Gill \\ Research Scholar \\ Department of Computer \\ Baba Farid College of Engineering And Technology, \\ Bathinda, Punjab, India
}

\author{
Er ChinuVerma \\ Assistant Professor: \\ Department of Computer \\ Baba Farid College of Engineering And Technology, \\ Bathinda, Punjab, India
}

\begin{abstract}
There are various non-driver related reasons for automobiles crashes including street conditions, the climate and the mechanical execution of automobiles. However, a significant number of automobile accidents are caused by driver error. Driver error includes drunkenness, fatigue, and drowsiness. Many factors can affect a driver's ability to control a motor vehicle, such as natural reflexes, recognition and perception. The diminishing of these factors can eventually reduce a driver's vigilance level. There have been numerous "raising awareness" campaigns about drowsiness and drunken driving. However, they have been ineffective for the most part. Such accidents not only affect the drowsy drivers, but also any potential victims. Driver Drowsiness Detection, which is used to detect or not a driver is drowsy can use different features i.e. heart rate, eye status etc. This work introduces an alerting process for when the driver fall asleep based on computer vision based mechanism in which eye status has been calculated by extracting frames from the ongoing video. There are various features which has been found in literature i.e. DWT, statistics, LBP etc. but still an improvement was required as the drowsiness detection results was not similar when different classifiers were used. Hence a contourlet transform and web local descriptor based feature set has been proposed which gives high accuracy in drowsiness detection when different classifiers have been tested by this feature set. Also video compression does not affect much when we evaluated this feature set and high accuracy has been achieved by different classifiers
\end{abstract}

Keywords: Contourlet transform, Drowsiness, fatigue, compression.

\section{INTRODUCTION}

Drowsiness is basically characterized as "a condition of close rest because of weariness". It is actually unmistakable from weakness, which has been characterized as an "unwillingness to keep playing out the job that needs to be done". The impacts of languor and weariness are almost the same. Exhaustion influences mental readiness, diminishing a person's capacity to work a vehicle securely and expanding the danger of human blunder that could prompt fatalities and wounds. Languor moderates response time, diminishes mindfulness, and weakens judgment. Exhaustion and lack of sleep affect all transportation administrators (for instance: carrier pilots, truck drivers, and railroad engineers).In the two conditions, driver can't concentrate on essential errand of driving which may improve the probability of crash event. With the consistently developing movement conditions, this issue will additionally break down. Therefore, it is important to create driver readiness framework for mishap counteractive action [1].In [2] image processing method is utilized for drowsiness recognition. The framework ought to distinguish the condition of the driver as ahead of schedule as could be allowed and the false identification rate ought to be diminished. The framework utilizes Gabor ordinal measures so as to identify the face.A nonintrusive drowsiness recognition method is used eyetracking and image processing[3].Open CV's library is used to accept each frame and store it in an image structure that can easily be used for further processing [4]. Eye movements of 14 drivers is observedusing electrooculography (EOG) at the moving-base driving simulator of Mercedes Benz to assess driver drowsiness[5]. A module for automatic driver drowsiness detection based on visual information and Artificial Intelligence is used to locate, track and analyze both the driver's face and eyes to compute a drowsiness index to prevent accidents[6]. Both face and eye detection is performed by Haar-like features and AdaBoostclassifiers[7].

\section{LITERATURE SURVEY}

Cheng et al. [8]exhibit a nonintrusive laziness acknowledgment technique utilizing eye-following and picture handling. A hearty eye identification calculation is acquainted with address the issues caused by changes in light and driver act. Six measures are figured with level of eyelid conclusion, greatest conclusion length, and squint recurrence, normal opening level of the eyes, opening speed of the eyes, and shutting speed of the eyes.

Emam et al. [9] proposed integration between image mining technique and the developed real time drowsy eye detection architecture. They present Intelligent Drowsy Eye Detection using Image Mining (IDEDIM) system architecture.

G. Kong et. al. [10]presents visual examination of eye state and head posture (HP) for ceaseless checking of sharpness of a vehicle driver. Most existing ways to deal with visual discovery of non-ready driving examples depend either on eye conclusion or head gesturing points to decide the driver tiredness or diversion level.

Loonis et al. [11]propose a novel wise reconnaissance framework to gauge driver sleepiness in light of the Observer Rating of Drowsiness (ORD) show incorporated into prove hypothesis through combination of path and eye 
highlights. ORD is a subjective evaluation of sluggishness that is reflected in individuals' physical appearance, practices and peculiarities.

Majlis et al. [12] evaluate the use of electrooculogram (EOG) as an alternative to video-based systems in detecting eye activities caused by drowsiness. The EOG, which is the electrical signal generated by eye movements, is acquired by a mobile bio signal acquisition module and are processed offline using personal computer.

Chun et al. [13]presents visual investigation of eye state and head posture (HP) for ceaseless checking of sharpness of a vehicle driver. Most existing ways to deal with visual identification of non-ready driving examples depend either on eye conclusion or head gesturing points to decide the driver tiredness or diversion level.

Yang et al. [14] assess the execution of the most recent eye following situated in-vehicle weakness forecast measures. These measures are evaluated factually and by an arrangement technique in light of an extensive dataset of 90 hours of genuine street drives.

Oguri et al. [15] presents a novel method that uses eyelid closure and heart rate variability to estimate the driver's drowsiness level. Laboratory experiments were conducted by using a proprietary driving simulator, which induced drowsiness among the test drivers.

Bhuiyan et al. [16]present an improved method for estimating a driver's drowsiness level using eyelid closure and heart rate variability. Laboratory experiments were conducted by using a proprietary driving simulator, which induced drowsiness among the test drivers.

Majlis et al. [17] evaluate the use of electrooculogram (EOG) as an alternative to video-based systems in detecting eye activities caused by drowsiness. The EOG, which is the electrical signal generated by eye movements, is acquired by a mobile bio signal acquisition module and are processed offline using personal computer.

\section{PROPOSED WORK}

The system module of the proposed system is shown in Figure 1. First of all, frames have been extracted from the captured video. Next eye region has been localized using cascade object detector. The cascade object detector uses the Viola-Jones algorithm to detect people's faces, noses, eyes, mouth, or upper body. Next a set of features are extracted from each eye patch to help discriminate between open and closed eye patches and used to build a Feature vector. Given an image feature set, different classifiers are employed to classify the images into drowsiness and non-drowsiness frame. The steps used are given below in figure 1 .

\section{- Local Binary Patterns}

The local binary pattern is a powerful gray level invariant texture primitive [18]. The histogram of the binary patterns computed over a region is used for texture description [19].

\section{- Contour Let Transform}

Most of the classical frequency domain transforms such as Fourier transform, Discrete Cosine Transform and Wavelet trans-form are very efficient in capturing the details as far as they are exploited in onedimensional context. In order to extract the details of an image, 2-D extensions are constructed from the 1-D separable basis functions. Such transforms are good at capturing the details when the image is a collection of 1-D piece-wise smooth signals.

\begin{tabular}{|c|}
\hline Acquire captured video and convert it to frames \\
\hline$\vee$ \\
\hline Patch extraction by Acquiring eyes as ROIs \\
\hline$\vee$ \\
\hline $\begin{array}{l}\text { Contourlet-WLD and LBP implementation and } \\
\text { generation of histograms }\end{array}$ \\
\hline$\vee$ \\
\hline $\begin{array}{c}\text { Labeling of training images based on two classes as } \\
\text { oDen and closed }\end{array}$ \\
\hline$\vee$ \\
\hline Concatenation of features into Matrix \\
\hline$\vee$ \\
\hline Classification using ANN/ kNN/SVM/C50 classifiers \\
\hline$\vee$ \\
\hline $\begin{array}{c}\text { Performance evaluation in terms of accuracy rate of } \\
\text { classification }\end{array}$ \\
\hline
\end{tabular}

Figure 1: System Module

\section{- Weber Local Descriptors (WLD)}

Weber's law signifies the relationship between incremental threshold ( $\Delta F$ ) and surrounding intensity $(F)$. It states that the ratio $\Delta \mathrm{F} / \mathrm{F}$ is always constant and denoted by Weber fraction [20] where, $F$ is initial intensity in the signal/image and $\mathrm{F}$ is the smallest change which is noticeable. For example, in signal processing context, in a crowded room, one has to speak loud in order to get heard, whereas a whispering sound is sufficient in a quiet place to get noticed. Such a phenomenon in the context of human vision is known as 'Just Noticeable Difference' (JND). Chen et al. [20] proposed the JND based Weber local descriptor (WLD) which became popular feature extraction technique for texture classification, face detection and face recognition. In particular, it leads to a representation close to biological human vision. The WLD histogram is constructed after mapping of differential excitation and orientation components. Further, it is projected onto 1-D space for classification efficiency.

\section{RESULT AND DISCUSSION}

The receiver operating characteristic is a metric used to check the quality of classifiers. For each class of a 
classifier, ROC applies threshold values across the interval $[0,1]$ to outputs. For each threshold, two values are calculated, the True Positive Ratio (TPR) and the False Positive Ratio (FPR). For a particular class i, TPR is the number of outputs whose actual and predicted class is class i, divided by the number of outputs whose predicted class is class $\mathrm{i}$. FPR is the number of outputs whose actual class is not class $i$, but predicted class is class $i$, divided by the number of outputs whose predicted class is not class $i$.

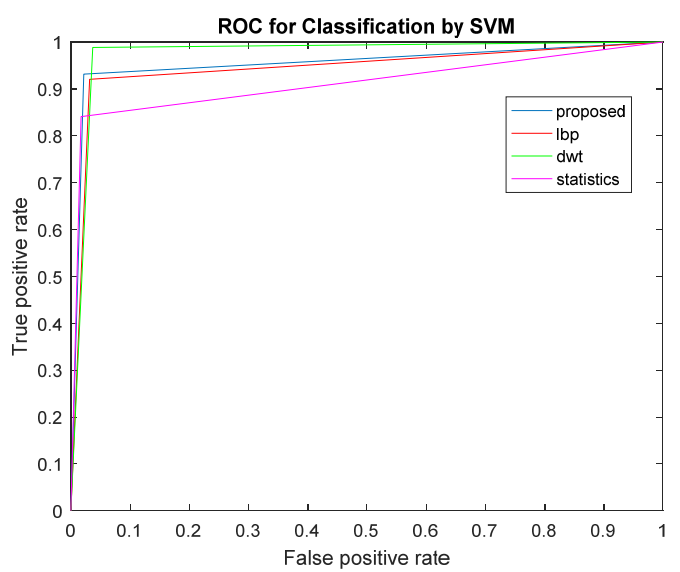

Figure 2: Receiver Operating Characteristics using SVM

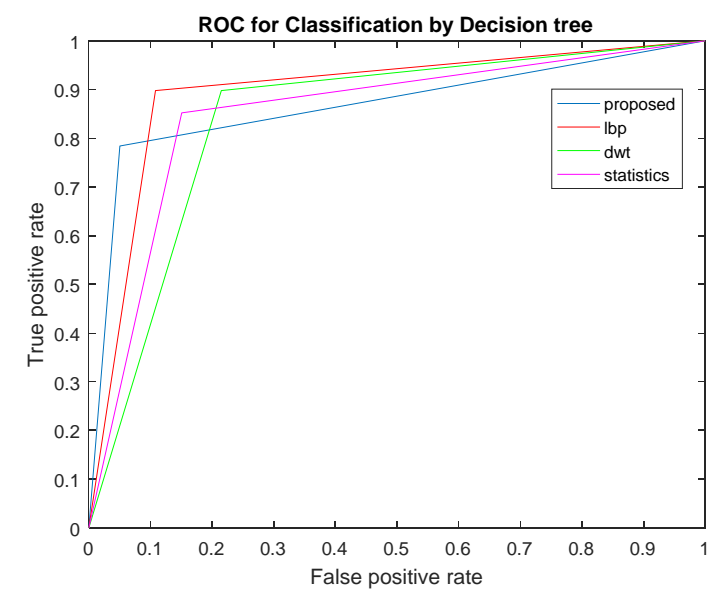

Figure 3: Receiver Operating Characteristics using Decision tree classifier

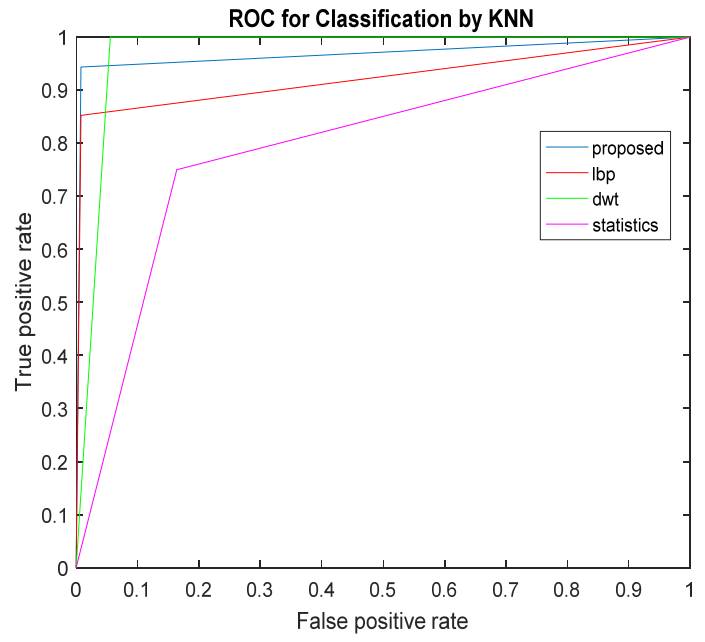

Figure 4: Receiver Operating Characteristics using KNN

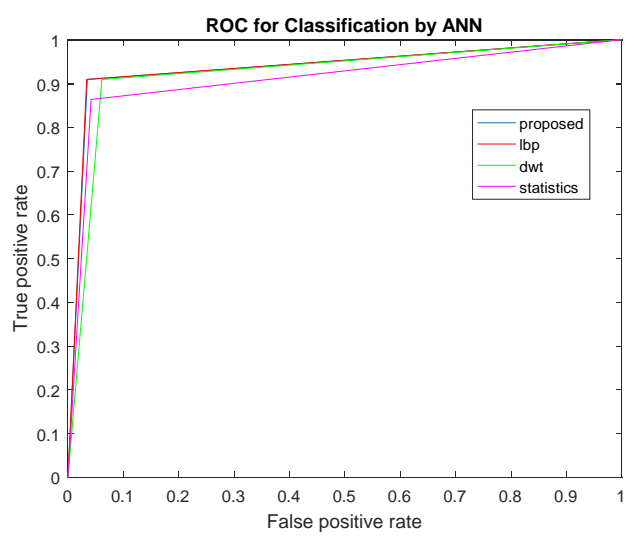

Figure 5: Receiver Operating Characteristics using ANN

The results for the actual pixel location using ground truth images and that of resulted outputs has been described with above parameters.

Table 1: Results in tabular form using different features and classifiers

\begin{tabular}{|l|l|l|l|l|}
\hline \multirow{2}{*}{$\begin{array}{l}\text { Classifier } \\
\text { used }\end{array}$} & \multicolumn{4}{|l|}{ Features used } \\
\cline { 2 - 5 } & LBP & DWT & Statistics & $\begin{array}{l}\text { Contourlet- } \\
\text { WLD and } \\
\text { LBP }\end{array}$ \\
\hline SVM & 96.4143 & 96.5139 & 97.0120 & 97.4104 \\
\hline $\begin{array}{l}\text { Decision } \\
\text { tree }\end{array}$ & 89.2430 & 79.4821 & 84.9602 & 93.5259 \\
\hline KNN & 98.0080 & 94.9203 & 82.8685 & 98.8048 \\
\hline ANN & 96.1155 & 93.6255 & 95.0199 & 96.0159 \\
\hline
\end{tabular}




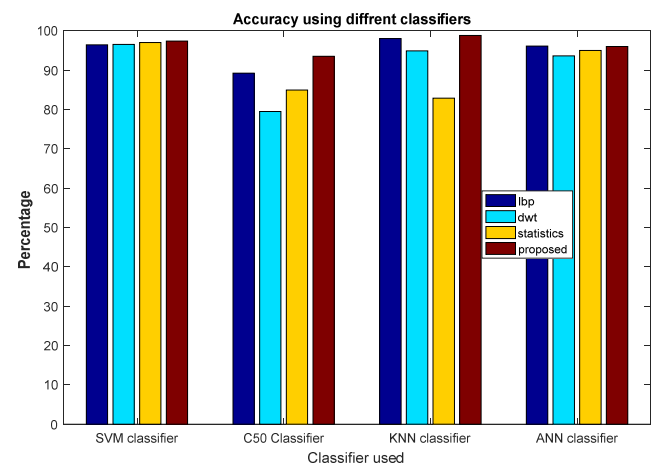

Figure 6: Classification Accuracy of different classifiers

Table 2: Results of classification after $50 \%$ video compression

\begin{tabular}{|l|l|l|l|l|}
\hline \multirow{2}{*}{$\begin{array}{l}\text { Classified } \\
\text { used }\end{array}$} & \multicolumn{4}{|l|}{ Features used } \\
\cline { 2 - 5 } & LBP & DWT & Statistics & $\begin{array}{l}\text { Contourlet- } \\
\text { WLD and } \\
\text { LBP }\end{array}$ \\
\hline SVM & 96.6056 & 96.0159 & 95.9163 & 97.4004 \\
\hline $\begin{array}{l}\text { Decision } \\
\text { tree }\end{array}$ & 92.7291 & 91.6335 & 91.1355 & 93.5159 \\
\hline KNN & 97.1116 & 96.6135 & 83.1673 & 98.8040 \\
\hline ANN & 95.1076 & 90.5378 & 95.3187 & 96.0150 \\
\hline
\end{tabular}

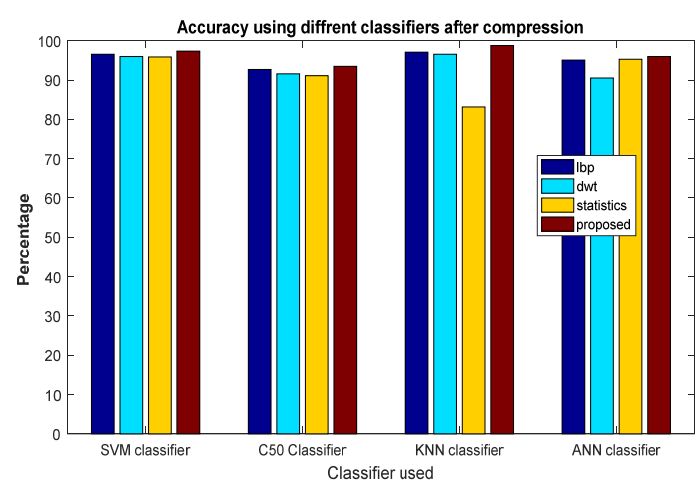

Figure 7: Classification Accuracy of different classifiers after compression

As shown in above table and graphs, there is not much difference in classification parameters when video has been compressed to $50 \%$. However if we increase the compression ratio, results can be degraded due to small size of the patches.

\section{CONCLUSION}

Driver drowsiness was perceived as a vital reason in the vehicle mishaps. It is demonstrated that driving performance reduces with increase in drowsiness. Advanced innovation offers some would like to maintain a strategic distance from these sorts of mischances up to some degree. As video camera facility is easily available, a computer vision oriented mechanism can gives real time results in alerting the driver when he/she feels sleepy based on the status of the eyes. Similar research has been explored in this work in which different types of features has been used on eye region. At first face has been detected from whole frame and later left and right eye patches has been extracted so that better features can be obtained only from eye region. Later a set of features has been evaluated in which LBP, statistics and DWT features has been used from existing literature and a new feature based on contourlet transform and web local descriptors has been introduced. Different classifiers i.e. ANN, SVM, decision tree etc. has been trained and tested using the extracted features from both closed and open eye datasets. Experimental results show high accuracy in drowsiness detection when new feature has been considered as compared to existed feature sets.

\section{REFERENCES}

[1]A.N.Shewale, PranitaChaudhari,"Real Time Driver Drowsiness Detection system." Published in: International Journal of Advanced Research in Electrical, Electronics and Instrumentation Engineering Vol. 3, Issue 12, December 2014

[2]A. Mary Sefia; J. AnithaGnanaSelvi,"Driver State Analysis And Drowsiness Detection Using Image Processing" Published in: International Journal of Scientific Engineering and Applied Science (IJSEAS) - Volume-2, Issue-6, June 2016.

[3]Wei Zhang, Bo Cheng, Yingzi Lin, "Driver Drowsiness Recognition Based on Computer Vision Technology" Published in Tsinghua Science and Technology (Volume:17 , Issue: 3 ) Page(s): 354 - 362 Date of Publication : June 2012.

[4]Bradski, G., the Open CV Library. Dr. Bobb's Journal of Software Tools, 2000.

[5]ParisaEbrahim, Wolfgang Stolzmann and Bin Yang, "Eye Movement Detection for Assessing Driver Drowsiness by Electrooculography" Published in Systems, Man, and Cybernetics (SMC), 2013 IEEE International Conference on Date of Conference: 13-16 Oct. 2013 Page(s): 4142 4148.

[6]MehrdadSabet, Reza A. Zoroofi, KhosroSadeghniiatHaghighit, Maryam Sabbaghian, "A New System for Driver Drowsiness and Distraction Detection” Published in Electrical Engineering (ICEE), 2012 20th Iranian Conference on Date of Conference: 15-17 May 2012 Page(s): 1247 - 1251.

[7]. Freund, Y. and R. Schapiro, "A short introduction to Boosting" Published in: Japanese Society for Artificial Intelligence, 1999. 14(5): p. 771-780.

[8]M. Sabet, R. A. Zoroofi, K. Sadeghniiat-Haghighi and M. Sabbaghian, "A new system for driver drowsiness and distraction detection," 20th Iranian Conference on Electrical Engineering (ICEE2012), Tehran, 2012, pp. 1247-1251

[9]Ahmed Emam," Intelligent drowsy eye detection using image mining," published in Springer nformation Systems Frontiers August 2015, Volume 17, Issue 4, pp 947-960

[10]Ralph OyiniMbouna, Seong G. Kong, Senior Member, IEEE, and Myung-Geun Chun," Visual Analysis of Eye State and Head Pose for Driver Alertness Monitoring.” IEEE Transactions on Intelligent Transportation Systems, Vol. 14, No. 3, September 2013.

[11]Xuanpeng Li, Emmanuel Seignez, and Pierre Loonis, "Vision-based Estimation of Driver Drowsiness with ORD Model Using Evidence Theory" Published in Intelligent 
Vehicles Symposium (IV), 2013 IEEE Date of Conference: 23-26 June 2013 Page(s): 666 - 671.

[12]Thum Chia Chieh, M. M. Mustafa, A. Hussain, S. F. Hendi and B. Y. Majlis, "Development of vehicle driver drowsiness detection system using electrooculogram (EOG)," 2005 1st International Conference on Computers, Communications, \& Signal Processing with Special Track on Biomedical Engineering, Kuala Lumpur, Malaysia, 2005, pp. 165-168

[13]Ralph OyiniMbouna, Seong G. Kong, and Myung-Geun Chun, "Visual Analysis of Eye State and Head Pose for Driver Alertness Monitoring” Published in IEEE TRANSACTIONS ON INTELLIGENT TRANSPORTATION SYSTEMS, VOL. 14, NO. 3, SEPTEMBER 2013.

[14]FabianFriedrichs and Bin Yang, "Camera-based Drowsiness Reference for Driver State Classification under Real Driving Conditions" Published in Intelligent Vehicles Symposium (IV), 2010 IEEE Date of Conference: 21-24 June 2010 Page(s): $101-106$.

[15]AyumiTsuchida, Md. ShoaibBhuiyan and Koji Oguri, "Estimation of Drowsiness Level Based on Eyelid Closure and Heart Rate Variability" Published in Engineering in Medicine and Biology Society, 2009. EMBC 2009. Annual International Conference of the IEEE Date of Conference: 3-6 Sept. 2009 Page(s): 2543 - 2546.
[16]AyumiTsuchida, Md. ShoaibBhuiyan and Koji Oguri, "Estimation of Drivers' Drowsiness Level using a Neural Network Based 'Error Correcting Output Coding' Method” Published in Intelligent Transportation Systems (ITSC), 2010 13th International IEEE Conference on Date of Conference: 19-22 Sept. 2010 Page(s): 1887 - 1892.

[17]Thurn Chia Chieh, Mohd.Marzuki Mustafa, AiniHussain, SeyedFarshadHendi, BurhanuddinYeopMajlis, "Development of Vehicle Driver Drowsiness Detection System Using Electrooculogram (EOG)" Published in Computers, Communications, \& Signal Processing with Special Track on Biomedical Engineering, 2005. CCSP 2005. 1st International Conference on Date of Conference: 14-16 Nov. 2005 Page(s): 165 - 168.

[18] Mahdi Hariri and FahimeHakimi "Image-Splicing Forgery Detection Based On Improved LBP and K-Nearest Neighbors Algorithm” Published in: Electronics Information \& Planning, 2015, 3(0304-9876), p. 7

[19]ManishaVerma, Balasubramanian Raman, "Center symmetric local binary co-occurrence pattern for texture, face and bio-medical image retrieval" Published in J. Vis. Commun. Image R. 32 (2015) 224-236

[20] Li, Y., Shan, S., Zhang, H., Lao, S., Chen, X.: "Fusing magnitude and phase features for robust face recognition" Published in: Proc. Asian Conf. on Computer Vision (ACCV12), Daejeon, 2012, pp. 601-612 